\title{
Laser scanning and CAD conversion accuracy correction of a highly curved engineering component using a precision tactile measuring system
}

\author{
Athanasios Giannelis ${ }^{1}$, Ioanna Symeonidou ${ }^{2}$, Dimitrios Tzetzis ${ }^{2, *}$, George Kaisarlis ${ }^{3}$, and \\ Panagiotis Kyratsis ${ }^{4}$ \\ ${ }^{1}$ Qcontrol, GR 17675, Athens, Greece \\ ${ }^{2}$ International Hellenic University, 14km Thessaloniki - N. Moudania, Thermi GR57001, Greece \\ ${ }^{3}$ National Technical University of Athens, School of Mechanical Engineering, Athens, GR15780, \\ Greece \\ ${ }^{4}$ Western Macedonia University of Applied Sciences, Department of Mechanical Engineering and \\ Industrial Design, Kila Kozani GR50100, Greece
}

\begin{abstract}
Impellers are used in various mechanical applications and they usually operate under severe conditions. Very often a replacement of an impeller is required after certain operation hours due to material failure. Since it is a critical and expensive spare part, accurate manufacturing, following the design requirements of the part, is necessary. In order to avoid any dimensional manufacturing defects, quality control procedures are used based on reverse engineering. The aim of the current paper was to evaluate the overall accuracy of the reverse engineering procedure, when a laser scanner is used for digital data capturing. The specified accuracy of the laser scanner was of $\pm 0.084 \mathrm{~mm}$ and the scanned data were converted to a Computer Aided Design (CAD) file using commercial design software. In order to assess the accuracy of the laser scanning and the CAD conversion procedure, a highly accurate coordinate measuring machine was used with a touch trigger probe and specified accuracy of $\pm 0.006 \mathrm{~mm}$. The results have shown that the deviation produced by the laser scanning and the associated reverse engineering methodology was up to $1 \mathrm{~mm}$, mostly observed at the edges of the freeform surfaces. It was concluded that such large deviations are caused from the inaccuracy of the laser scanner, possible errors created by the mesh - polygonal model creation, but most importantly from the errors when creating the B-splines.
\end{abstract}

\section{Introduction}

Reverse Engineering (RE) is the process of discovering the technological principles of an object or component through analysis of its structure and function [1]. It is very often used in mechanical parts for a variety of reasons, which may relate to the availability of a spare

\footnotetext{
* Corresponding author: d.tzetzis@ihu.edu.gr
} 
part or in order to redesign and improve the function of the spare part [2]. There is a great range of techniques, which are all accompanied by their advantages and limitations. Most of the RE techniques include three main phases, namely a) data capturing, b) data processing (point cloud processing, triangulation, segmentation, alignment, meshing) and c) downstream applications i.e. CAD model, prototyping [3, 4].

The present research aims to analyse the advantages and the disadvantages of the procedures applied when creating a 3D model, measure and evaluate the errors formed during different stages of the process, study the accuracy of the reverse engineering procedures and detect any weaknesses in order to propose improvements of the process. An impeller is chosen for the evaluation since often it requires replacement after a certain number of operation hours or after material failure.

For the RE of the impeller a laser scanner was used and evaluated with respect to its accuracy and ease of use. For the evaluation, a coordinate measuring machine (CMM) with touch trigger probe, which is basically a tactile measurement method with a high accuracy, was used as well. With the CMM the part was re-measured and the measured data were compared to the created 3D CAD model with the use of specialized measuring software.

\section{Laser scanning procedure}

The laser scanner used was the ROMER Absolute Arm 7-Axis from the "SI" series, a configuration that requires no external controller box and has good characteristics, ergonomics and ease of use. Its automatic laser power control allows scanning of different surface types [5].

The operation of the scanner is manual and therefore it has to be manually driven over the surfaces which are to be scanned. The mounting of the part must safeguard that it shall not be moved during the scanning process. Through a set of absolute encoders the system recognizes the position of the scanner and the angle to the part. For the data collection and the data acquisition InnovMetric PolyWorks ${ }^{\mathrm{TM}}$-Standard was used, as it offers a range of benefits related to mesh generation, sketching and file format compatibility. The process was divided in certain steps using a variety of tools: a) IMAlign ${ }^{\mathrm{TM}}$ - collecting and editing the point cloud, b) IMMerge ${ }^{\mathrm{TM}}$ - creating the polygon file, c) IMEdit ${ }^{\mathrm{TM}}$ - edit the polygons and creating the curves \& NURBS, d) IMInspect ${ }^{\mathrm{TM}}-$ measuring software.

\section{Coordinate measuring machine}

For the evaluation of the performed methodology, a system with a higher accuracy is required. With this system the deviations are measured between the created 3D model and the real object. For selecting an appropriate measuring system the commonly-used "Rule of Ten" was followed, i.e. the measuring instrument should be 10 times more accurate than the characteristic to be measured. The whole idea was to choose an instrument that was capable of detecting the amount of variation present in a given characteristic. Since the certified scanning system accuracy of the laser scanner was $\pm 0.084 \mathrm{~mm}$, the measuring system used for the evaluation should offer a system accuracy of less than $\pm 0.009 \mathrm{~mm}$. For measuring the object in order to perform a CAD to part comparison, the CMM DEA/Brown \& Sharpe/Mistral with PH10M/TP200 Renishaw head was used (measuring range: $700 \mathrm{mmx} 700 \mathrm{~mm} \times 500 \mathrm{~mm}$, specified measuring accuracy according to ISO $10360-2$ : $\mathrm{U}(\mu \mathrm{m})$ $=2.5+\mathrm{L}(\mathrm{mm}) / 250$, where $L$ is the measured length). For the evaluation the measuring software PC-DMIS CAD++ was used, since it was state-of-the-art metrology software that effectively uses CAD models for the task of inspecting and evaluating parts. 


\section{Reverse engineering workflow}

\subsection{Scanning and data collection}

When setting up the scanning workstation, the area of the part used for mounting was kept to a minimum. For the scanning procedure, the impeller was placed on the side of a granite table on which the scanner was also mounted and stabilized. Polyworks ${ }^{\mathrm{TM}}$ IMAlign $^{\mathrm{TM}}$ was used for digitizing the part and for collecting the point cloud data. A plug-in tool enabled the direct interface with the laser scanner and the real time visualization of the digitalization process.

The laser density is automatically adjustable according to the part's surface colour and gloss. The point cloud is organized in a grid whose spacing was set by the parameters max edge length and interpolation step. The interpolation step used for this particular set-up was $0.3 \mathrm{~mm}$ and the max edge length was set at the $10 \mathrm{x}$ the interpolation step. The maximum angle parameter was adjusted to $70 \mathrm{deg}$. for providing sufficient points to reconstruct the surface. The outcome was several clouds of points (highlighted in different colours - see Figure 1). Since only the upper part of the impeller was totally accessible for the scanner laser stripe, the scanned data included several gaps that appear as holes. In Figure 1, the non-closed scans appear blue, as these are the scanned surfaces from the inside.
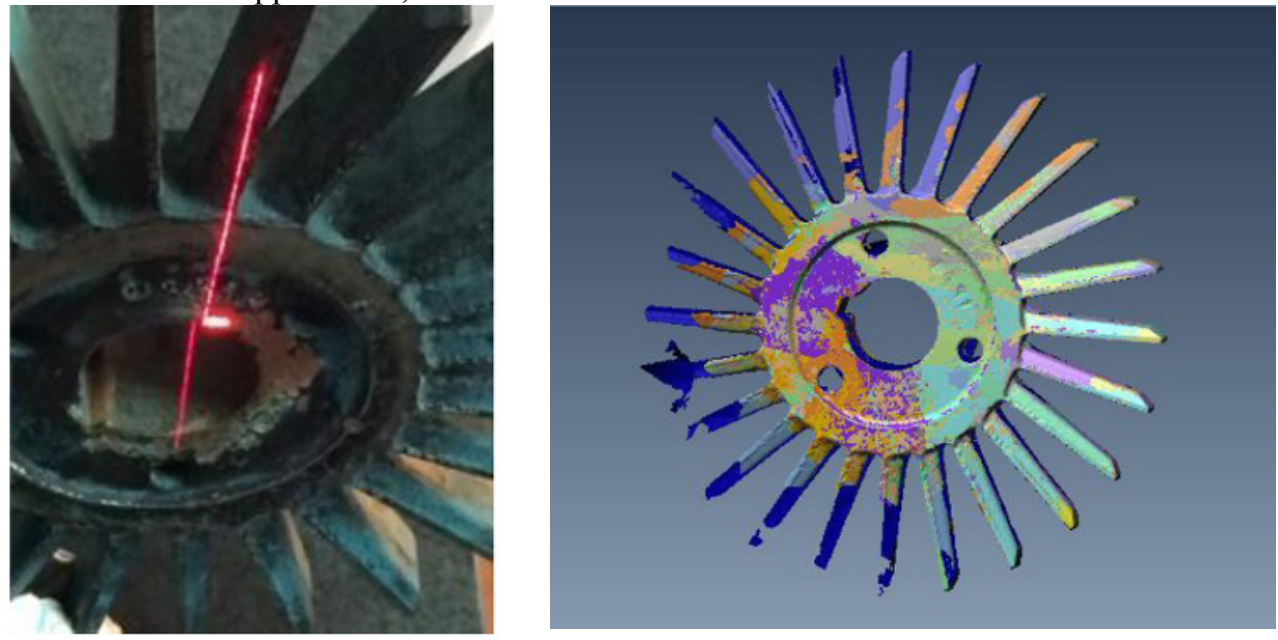

Fig. 1. The scanned object and the corresponding point clouds.

\subsection{Processing of the point clouds}

The created cloud of points is a cluster of different sub-clouds. Every time a new start point was used a new grid of points was created. This was used in order to log the different scan directions, which is helpful for further point processing. These several scans were overlapped in several areas (Figure 2). For the impeller used, 84 scans were performed.

The next action was to remove captured noise from reflection and fixtures, as well as points, which were not required for further processing. For creating the polygon- mesh and proceed with the CAD creation, it was recommended to reduce the size of the file. The IMAlign $^{\mathrm{TM}}$ (Figure 3 ) reduced the cloud of points by reducing the overlapping points and by keeping only two overlapping clouds of points in areas where the scans were coincident. 


\subsection{Creating the polygons (stl) and the curves (B-splines)}

The polygon mesh creation procedure was performed automatically and the parameters were predefined by the grid settings. For processing the polygon mesh, the IMEdit ${ }^{\mathrm{TM}}$ tool was employed. It was possible to optimize, smooth and reconstruct the polygon mesh and also to reconstruct surfaces in order to close the holes. In the process of transforming the point clouds into polygonal surfaces, holes appeared in the areas, where no points were captured. For optimizing the mesh, the polygons were reconstructed so that the angles, sizes and vectors were as uniform as possible. Smooth and reconstruct were not considered at this stage, as they would change the original surface data. Automatic hole filling was used for small holes, based on curvature calculation, while bigger holes were evaluated by the user via an interactive procedure. By completing the aforementioned steps, a watertight mesh was created, which was suitable for further digital fabrication.

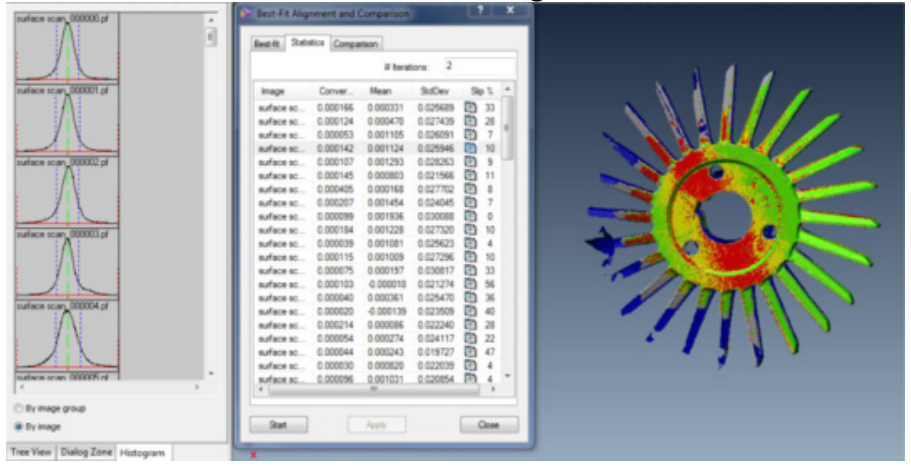

Fig. 2. A best fit alignment aligns the scans on the overlapping areas.
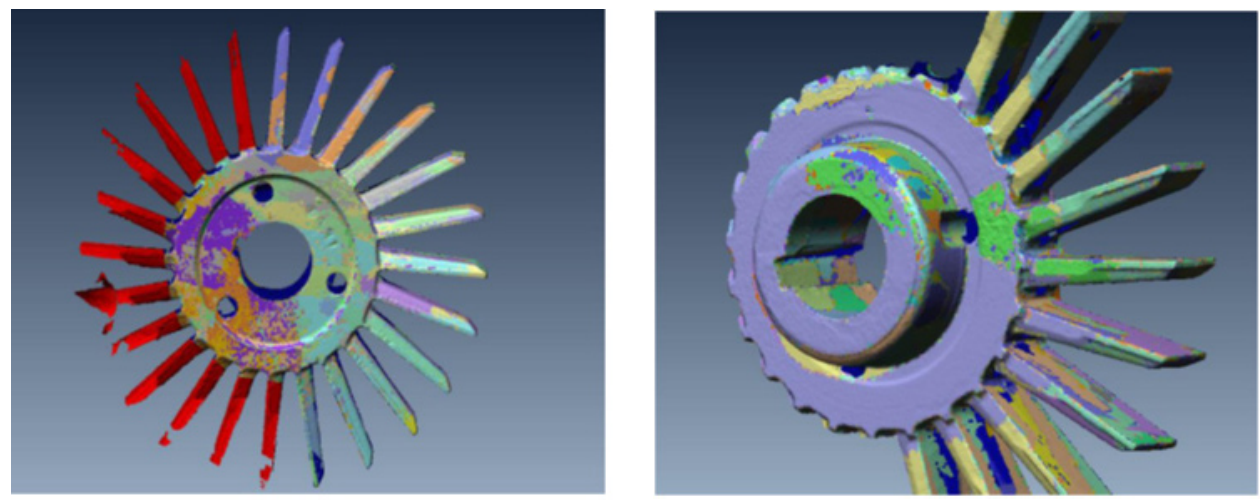

Fig. 3. Cleaning and reducing the cloud data.

Among the possible options for reconstructing a model, it was decided to create the curves and export them to a CAD system. The data could be imported in the CAD system and used directly to extract the features of the model. For the rapid reconstruction of the model one single blade was considered enough, as this could be circularly patterned. All the created sketches were exported as an iges file, which is a universal file format and was imported in the CAD system (Figure 4). Planes were created based on sketches. Then these 2D features were converted into 3D features by using several of the available CAD tools. Geometric and freeform features were used to create the core base and the blades respectively. 


\subsection{Measurements with a tactile measuring system}

Matching the CAD model coordinate system with the part coordinate system is a primary, yet rather delicate CMM task. For this work, a typical feature-based (plane/circle/circle) coordinate system was chosen and the potential errors were considered a priori. PC-DMIS software aligned CAD and part coordinate systems. The CMM was then set in DCC mode to automatically take over scanning procedure by: (i) generating the theoretical CMM paths for sets of points on repetitive scanning patterns, through the use of "Linear Open Scan" tool, (ii) capturing representative single points by the "Auto Vector Point" option. The $\mathrm{CMM}$ probe head was driven by the $\mathrm{X}, \mathrm{Y}, \mathrm{Z}$ coordinates of the selected $\mathrm{CAD}$ features and random points, which should be the same with the coordinates of the actual part. The real coordinates were recorded and the deviation was calculated (range: max $=0.94 \mathrm{~mm}$, $\min =0.031 \mathrm{~mm}$ ). This range was confirmed after several experimental iterations, thus one cylindrical feature and 87 measuring points were considered representative.
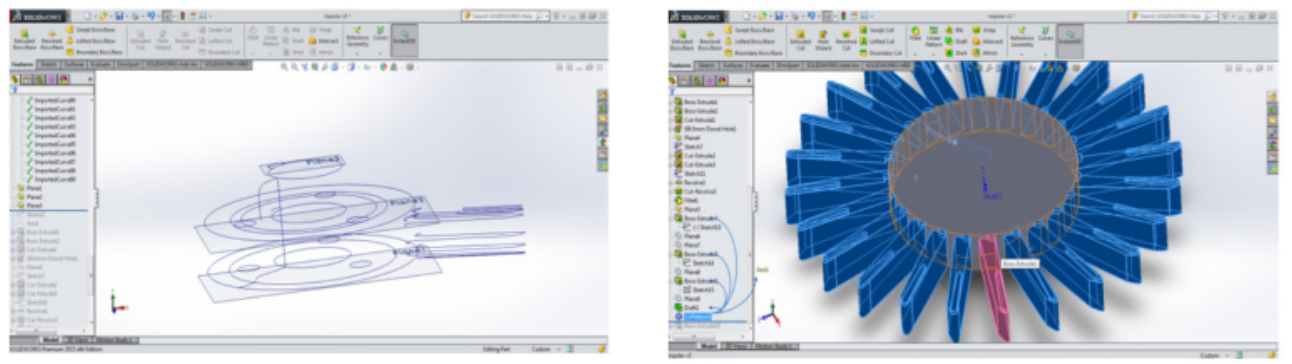

Fig. 4. Importing the data in SOLIDWORKS ${ }^{\mathrm{TM}}$ and creating the blades through circular pattern.

\subsection{Measurements with a laser scanner}

The second part of the evaluation of the accuracy of the reverse engineering procedure was to compare the convergence with the laser scanned data to the created CAD file. For this, the Polyworks ${ }^{\mathrm{TM}}$ software offers a measurement plugin named IMInspect ${ }^{\mathrm{TM}}$. The CAD file and the cloud of points were imported and aligned to each other. Then the deviation of the scanned points to the surfaces was measured. The software calculated automatically the deviation of all the measuring points to the nominal data; this was visualized on the CAD screen.

\section{Data analysis}

The aim of this research was to examine the accuracy of the reverse engineering procedure of creating 3D models from scanned data for mechanical parts with freeform surfaces. For this reason the measuring software's Polyworks ${ }^{\mathrm{TM}}$ and PC-DMIS were used. In both cases, the measured points with the tactile system and with the laser scanner were compared to the nominal values of the $\mathrm{CAD}$ data. In the examined case, the following measurements were applied: 1. Comparison of the created 3D Model within the used CAD system with the points measured with the laser scanner within the Polyworks $\mathrm{IM}^{\mathrm{TM}}$ inspect software tool and 2. Comparison of the created 3D Model in the CAD software with the points measured with the tactile system of the CMM in the PC-DMIS software. With both measurement procedures it was observed that the max deviations were up to $1 \mathrm{~mm}$. These deviations were mostly monitored near the edges of the freeform surfaces (Figure 5). Even though the RE technology of hardware and software is continuously improving, the design process steps are still error-prone. The entire procedure is not yet fully automated and therefore the 
experience of the operators and designers is of crucial importance. During the whole procedure and by evaluating the data with several systems it was observed that the errors have been determined by the following causes: 1 . Laser scanning accuracy of $\pm 0.084 \mu \mathrm{m}$; 2. Unevenness of the impeller surfaces caused by the operation of the component; 3 . Errors created by the mesh - polygonal model creation; 4 . Errors created while generating the BSplines; 5. Selected curves are not representative enough for the part; 6. Taking into account one single blade for the pattern and other errors made by the designer in the design software; 7. Alignment Errors of the CAD data with the measured points of the CMM.
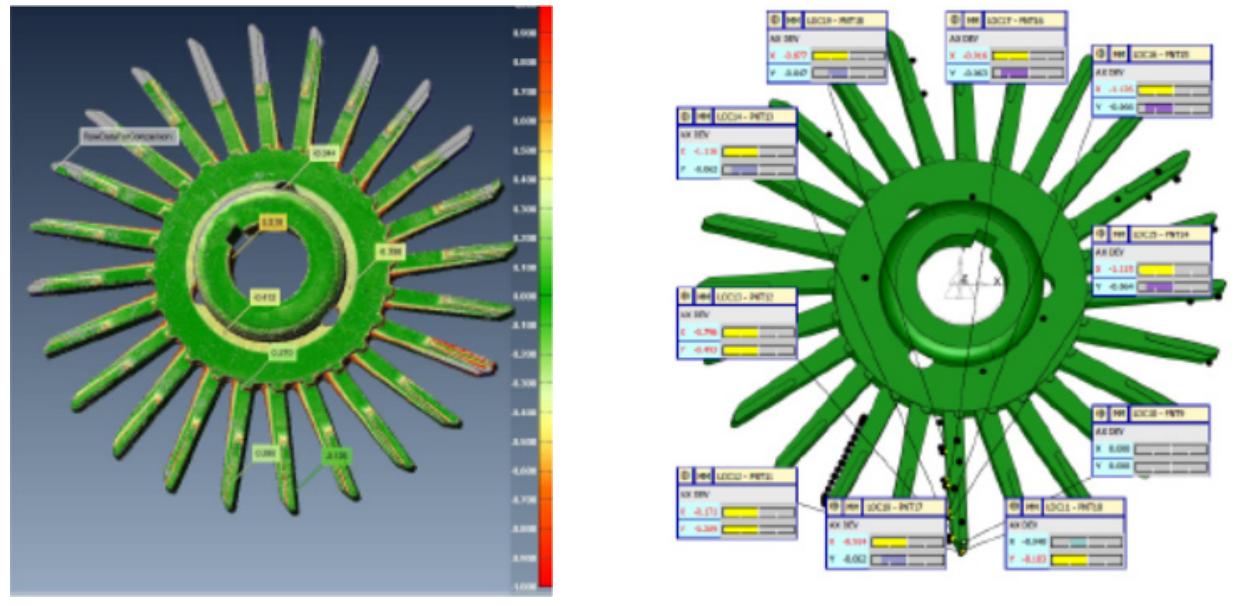

Fig. 5. The colour map scale showing the max and min values of the measured deviation.

\section{Conclusions}

The measurement results provide sound evidence that the technology is not ready yet to offer a fully-automated solution that safeguards the accuracy during reverse engineering of parts. Lack of user-friendly tools for the analytical, GUM-based expanded uncertainty estimation of the whole RE procedure is certainly a drawback. It could be possible to redesign the CAD model according to the measured deviations but the outcome is questionable and should be further examined. In any case, the technology provides certified accuracy of the measurement instrumentation which can, to a certain extent, ensure the designer about the quality of the RE procedure.

\section{References}

1. V. Raja, K. Fernandes, Eds., Reverse Engineering - An Industrial Perspective (Springer, 2010)

2. T. Varady, R.R. Martin, J. Cox, , Comput.-Aided Des., 29(4), 255-268 (1997)

3. K. Papadopoulos, D. Tzetzis, G. Oancea, P. Kyratsis, Academic Journal of Manufacturing Engineering, 14(1), 28-35 (2016)

4. G.R. Buican, G. Oancea, A. Manolescu, Applied Mechanics and Materials, 693, 285290 (2014)

5. "Romer Absolute Arm | SI." [Online]. Available: http://www.exactmetrology.com/metrology-equipment/romer-scanner/romer-absolutearm [Accessed: 11-Feb-2017] 